\title{
Biomimetic strain-sensing microstructure for improved strain sensor: fabrication results and optical characterization
}

\author{
D H B Wicaksono ${ }^{1}$, J F V Vincent ${ }^{2}$, G Pandraud ${ }^{1}$, G Craciun ${ }^{1}$ \\ and $\mathbf{P}$ J French ${ }^{1}$ \\ ${ }^{1}$ Delft University of Technology, DIMES-EI, Mekelweg 4, 2628 CD, Delft, The Netherlands \\ ${ }^{2}$ Bath University, ME, Bath BA2 7AY, UK \\ E-mail: d.h.b.wicaksono@ewi.tudelft.nl
}

Received 30 November 2004, in final form 25 May 2005

Published 20 June 2005

Online at stacks.iop.org/JMM/15/S72

\begin{abstract}
This paper describes the fabrication, and early optical characterization results of a new biomimetic strain-sensing microstructure. The microstructures were inspired from the campaniform sensillum, a highly sensitive strain sensor found in the exocuticle layer of insects. We investigate the natural strain-sensor characteristics by mimicking some of its simplest structural features. Blind-hole- and membrane-structural features were combined and fabricated as membrane-in-recess microstructure. To investigate the strain-sensing (or strain-amplifying) property of the microstructure, an optical characterization setup was devised based on the interference pattern formed by reflected laser beams from different surfaces of the microstructures. Preliminary qualitative analysis of the results obtained shows unsimilar intensity level changes as a function of spatial location on the membrane, thus indicated the biomimetic microstructure's strain-amplifying property. This property could be utilized for future improvement of currently available planar-based conventional strain sensors.
\end{abstract}

(Some figures in this article are in colour only in the electronic version)

\section{Introduction}

Nature has long been an inspiration for engineers. Many of today's world engineering masterpieces and work resemble those similar structures or systems found in nature [1-3]. Yet it was not until recently that the sensor engineering society started to take inspiration from high-performance sensors found in nature (see [4] for a comprehensive overview of current emerging development of nature-inspired sensors).

A campaniform sensillum (figure 1) is a kind of strain sensor found in insects, e.g. the blowfly (Calliphora vicina). The campaniform sensillum is basically an opening in the cuticle (with a size of 5-10 $\mu \mathrm{m}$ in diameter for the circular shape one) covered by membrane layers. The shape of the opening is generally ellipse and sometimes almost circular $[5,6]$. Deformation in the insect's cuticular layer is sensed by the campaniform sensillum using mechanical coupling, transduction and an encoding mechanism to transfer the environmental information to the insect's nervous system. Previous work by one of the authors (JFVV) [7] showed that the mechanical coupling mechanism was resolved into discrete components: a cap surrounded by a collar, a joint membrane and an annulus-shaped socket septum with a spongy compliant zone (the spongy cuticle). The coupling mechanism is a mechanical linkage which transforms the stimulus into two deformations in different directions: monoaxial transverse compression of the dendritic tip of a sensory neuron cell, which acts as a transducer, and vertical displacement of the cap. The natural campaniform sensilla, regardless of the high Young modulus of the exocuticle layer of the insect $(k \approx$ $10^{9} \mathrm{Nm}^{-2}$ ), can still detect changes. These sensors are as sensitive to displacement in that stiff structure as the receptors in the human ear are to sound [8]. This sensitivity is among others due to their unique membrane-in-recess microstructure. The membrane located inside a blind hole amplifies the strain. For a rather detailed discussion on the sensing mechanism, 


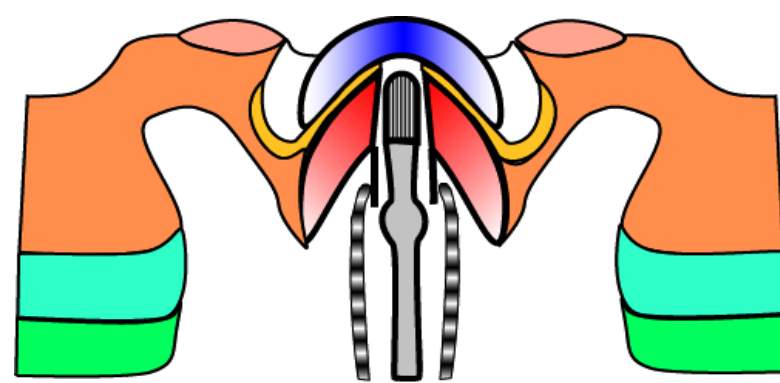

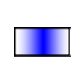

Cap

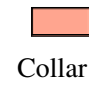

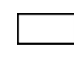

Joint Membrane

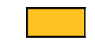

Spongy Cuticle
Exocuticle

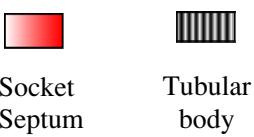

Mesocuticle

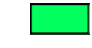

Endocuticle

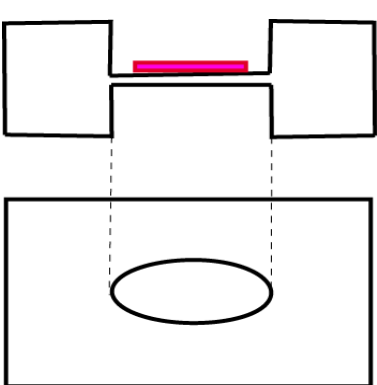

(a)

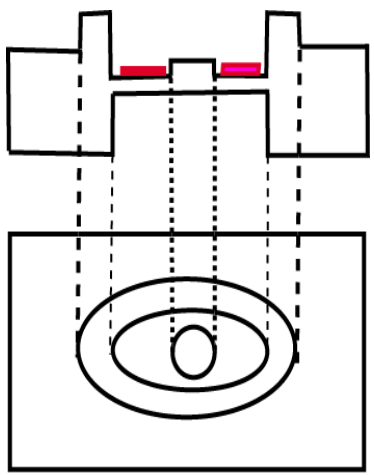

(c)

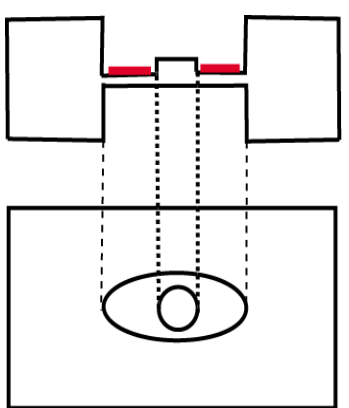

(b)

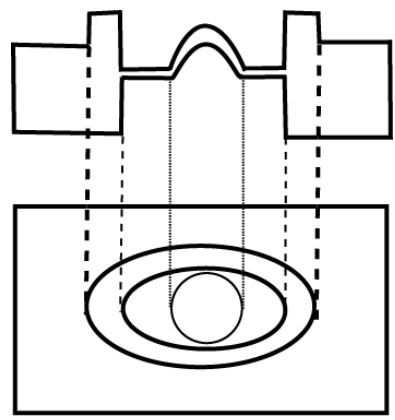

(d) The design and developint going research is illustrated briefly in figure 2 .

Membranes and sharp edges are well known to be used for increasing stress locally. The use of membranes and sharp edges (hole) combined in one structure for stand-alone strain sensing, however, is to the best of our knowledge still very limited. MEMS engineers have used membranes and diaphragms for detecting pressure, i.e. a vertical force applied perpendicular to the membrane, of which the stress on the edge of the membrane is further transduced with deposited piezoresistors. The use of holes as a stress-concentrating region has been reported to improve sensitivity in piezoresistive silicon cantilevers for scanning probe microscopy applications, as reported in [10-12].

In those reports, however, the holes were used as a component in cantilever sensors for application in biosensing or microscopy. Yet, the use of a (blind) hole to concentrate strain, which is transduced into a signal in a microstructure which is used as a stand-alone strain sensor, has never been reported before, to the best of our knowledge. Most of the papers in engineering mechanics did report characteristics of 'through hole' as stress concentrators and initial points of cracks, but not as an 'information collector' which is of interest for sensor engineers.

A MEMS-based strain sensor, which will be our next future step to apply this biomimetic strain-sensing microstructure for strain-sensing application, is advantageous compared to conventional thin metal foil strain gages and simple piezoresistive strain gages, in that it could provide higher sensitivity [13], as well as possibility for integration within a structure [14]. In the case reported in this paper, this better sensitivity will be expected to come from its unique membrane-in-recess structure that amplifies and concentrates strain, in comparison to direct transducing found in most available planar-structure strain sensors.

Figure 2. Design and development strategy and stages in imitating/mimicking natural campaniform sensillum strain sensor in a microelectronics-compatible MEMS processing scheme: (a) membrane-in-recess structure, employing blind-hole- and membrane-structural features, $(b)$ membrane-in-recess with additional 'cap'-like structure in the middle, $(c)$ as in $(b)$ but with additional 'collar'-like structure, $(d)$ a nearly complete mimics with dome-shaped 'cap'-like structure. See [9] for detailed discussion on these design stages.

This paper describes the continuation of our work [9, 15] to develop a MEMS-based strain sensor inspired from the campaniform sensillum of insects, especially those found in Calliphora vicina (the blowfly). This paper, however, will not describe a complete MEMS strain sensor with electrical read out. Rather, the goal of this paper is to investigate, through optical characterization, the micromechanical properties of the biomimetic membrane-in-recess structure. Then we intend to prove the indication that this structure, which is the first stage in our on-going research (figure 2), amplifies strain. An initial characterization of the mechanical properties based on real experimental results (in this case using optical methods) will be important to justify further addition of an electrical transducer onto the structure. That is why, in this paper, a direct comparison with available electrical strain sensors will not be carried out.

\section{Modeling and simulation}

To justify experimental investigation of strain-sensing microstructures inspired from the campaniform sensillum, we have previously reported modeling and numerical simulation of the natural campaniform sensillum [7]. In the twodimensional model, the campaniform sensillum was modeled as an opening in a fibrous plate. The opening was made 
Table 1. Simulation results indicated by bulk elongation and compliance of the openings under $1000 \mathrm{~N} \mathrm{~mm}^{-1}$ tensile load (adapted from [7]).

\begin{tabular}{|c|c|c|c|}
\hline Opening type & $\begin{array}{l}\text { Bulk } \\
\text { deformation }\end{array}$ & $\begin{array}{l}\text { Parallel to } \\
\text { the loading }\end{array}$ & $\begin{array}{l}\text { Normal to } \\
\text { the loading }\end{array}$ \\
\hline No opening & 2.85 & 2.87 & -0.87 \\
\hline Circular drilled & 2.91 & 11.90 & -5.90 \\
\hline Circular formed & 2.94 & 10.80 & -9.44 \\
\hline $\begin{array}{l}\text { Elliptical-drilled major } \\
\text { axis normal to load }\end{array}$ & 2.90 & 19.30 & -4.71 \\
\hline $\begin{array}{l}\text { Elliptical-formed major } \\
\text { axis normal to load }\end{array}$ & 2.92 & 15.81 & -7.01 \\
\hline $\begin{array}{l}\text { Elliptical-drilled major } \\
\text { axis parallel to load }\end{array}$ & 2.87 & 7.43 & -6.08 \\
\hline $\begin{array}{l}\text { Elliptical-formed major } \\
\text { axis parallel to load }\end{array}$ & 2.92 & 7.49 & -12.77 \\
\hline
\end{tabular}

either circular or elliptical, and either as drilled hole (i.e. the direction of the fiber is not effected by the hole) or formed hole (i.e. the fiber followed the edge of the hole). The plates were loaded with $1000 \mathrm{~N} \mathrm{~mm}^{-1}$ stress in one axis. The results of the $2 \mathrm{D}$ modeling and simulation are listed in table 1 . At the selected maximum load of $1000 \mathrm{~N} \mathrm{~mm}^{-1}$ the average tensile strains in the load direction range from $2.85 \%$ (no opening) to $2.94 \%$ (circular-formed hole). The openings amplify the local deformation up to eightfold, compared with the plates with no holes.

A three-dimensional model and simulation was also carried out based on pG4 campaniform sensillum [5, 6]. Different mechanical properties were allocated to the component of the sensillum, while all the cuticular materials are assumed to be isotropic. The axonometric view of the $3 \mathrm{D}$ finite-element model is shown in figure 3 . The model was subjected to arbitrary tensile and compressive stresses of $3 \mathrm{MPa}$. Another homogeneous model (i.e. with the same material properties for the whole structure) with similar structure was also made. The 3D finite-element modeling result is shown in figure 4. The figure indicates vertical displacement of the cuticular cap, and reveals that the cap indents (moving downward) under the tensile stress of the surrounding and bulges (moving upward) under the compressive stress. Further technical details and discussion about this modeling and numerical simulation of the natural campaniform sensillum as strain-sensing structure can be found in [7].

Based on the modeling results, further in this paper, an initial Si-based biomimetic structure will be fabricated using a microelectronics-compatible process, and its mechanical strain amplifying properties will be experimentally investigated using an optical method.

\section{Design and fabrication}

To investigate the strain-sensing property of the campaniform sensillum experimentally, we fabricated a membrane-in-recess structure. The structure is illustrated in figure 5 . The specificity of our design proposal shown in figure 5 lies in its combination of two structural features, previously only used separately: (1) blind-hole structure for concentrating strain, (2) 'buried' membrane, or membrane-in-recess structure for transforming the stimulus into two deformations in different

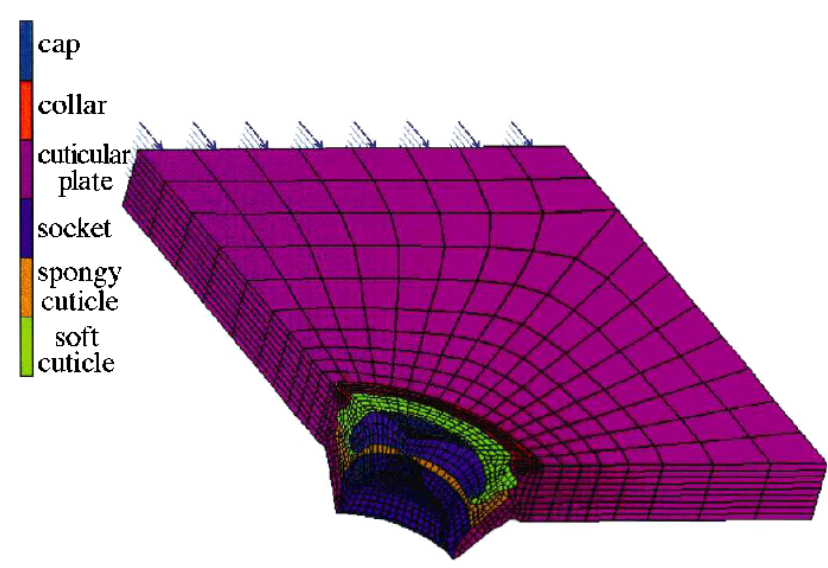

Figure 3. Axonometric view of 3D finite element of the pG4 campaniform sensillum (after [7]). The stress is applied along the top edge (dotted arrows).

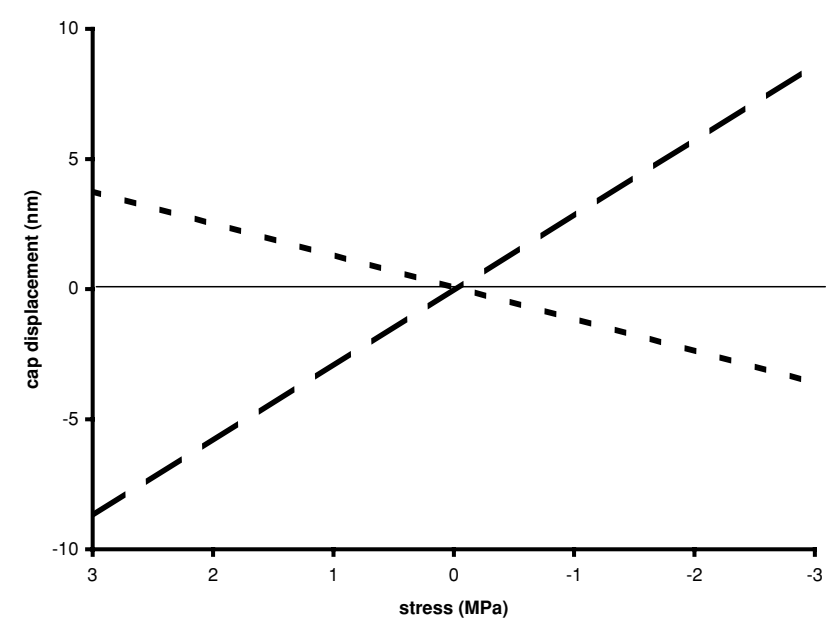

Figure 4. Output curves of the 3D finite-element model of the vertical displacement of the cap of the entire sensillum. Positive values of stress represent tensile stress, while negative values represent compressive stress. Positive values of cap displacement represent bulging, while negative values represent indentation. Long dashes: homogeneous model; short dashes: heterogeneous model (after [7]).

directions: transverse direction and vertical displacement. So, the strain to be detected comes from around the body of the structure, rather than from the vertically outside force, as commonly used in membrane-based pressure sensors. This combination of two structural features of the campaniform sensillum is our initial effort (see figure 2 and [9]) to develop a new strain sensor with better performance than currently available 'planar' strain sensors.

There are several parameters of interest that might have an influence on the strain-sensing property as illustrated in figure 3: $D$, the diameter of the hole; $h_{1}$, the depth of the hole and $t$, the thickness of the membrane. In this particular experiment, the membrane thickness was made constant at $500 \mathrm{~nm}$ as will be described later. The diameter of the circular membrane varies from $10 \mu \mathrm{m}$ to $1000 \mu \mathrm{m}$. At the moment, only membrane-in-recess with $h_{1}=13 \mu \mathrm{m}$ was fabricated. The processing yield before characterization for this membrane-in-recess structure is estimated to be around $60 \%$. 


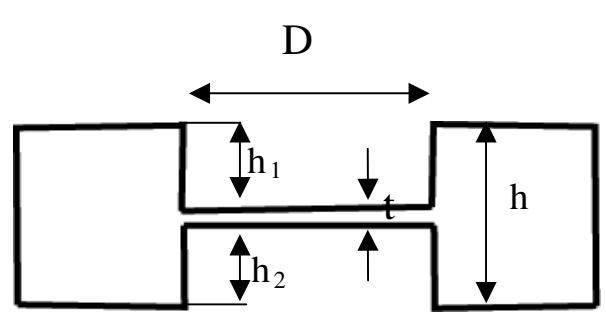

Figure 5. Membrane-in-recess structure as an initial biomimetic structure inspired from campaniform sensillum, $D$ : diameter of the hole, $t$ : membrane thickness, $h$ : thickness of Si-chip, $525 \mu \mathrm{m}$, $h_{1}$ : depth of the hole from top surface (defined by front-etching then deposition of $\mathrm{SiO}_{2} / \mathrm{SiN}$ thin films), $h_{2}$ : remaining thickness of the $\mathrm{Si}$ chip $=h-h_{1}-t$.

(a)

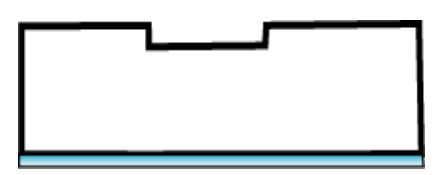

(b)

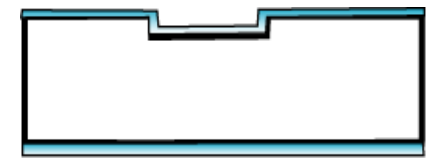

(c)

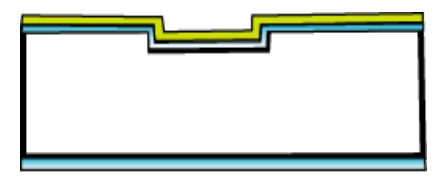

(d)

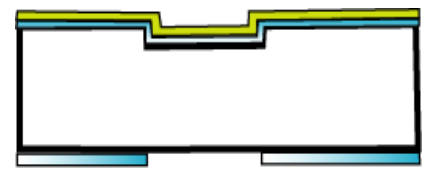

(e)

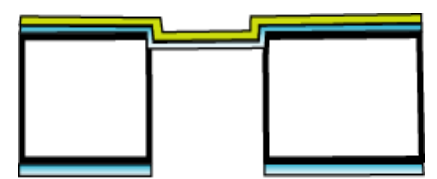

Figure 6. Process flowchart scheme for the two microstructures described in figure 2.

Figure 6 illustrates the fabrication flowchart scheme we used to produce the sample described in figure 5.

(1) Holes are first etched on the front side of a silicon wafer (figure 6(a)) using the inductively coupled plasma (ICP) etching process in a Trikon Omega 201 etcher. The aspect ratio achievable with reactive ion etching is low. In the following we limit the use of such a technique to etch the front surface of the wafers.

(2) $\mathrm{A} 200 \mathrm{~nm} \mathrm{SiO}_{2}$ was then thermally deposited (figure 6(b)). This layer will be used as the etch stop during the later back etching process.

(3) Finally a LPCVD $300 \mathrm{~nm}$ low stress SiN layer is deposited (figure 6(c)).
(4) On the backside of the wafers the oxide and nitride layers are etched away. Then an oxide mask layer is formed for further backside etching of the bulk Si wafer (figure 6(d)).

Then the backside of the structures was etched using the ICP etching process at $-105{ }^{\circ} \mathrm{C}$ (figure 6(e)). The experiments have been performed in a high density ICP reactor (Alcatel MET). We used the following gas concentrations to achieve good verticality angle: $\mathrm{SF}_{6} 400 \mathrm{sccm}$ and $12 \mathrm{sccm} \mathrm{O}$. Prior to the etching a cleaning of the chamber is performed using $100 \mathrm{sccm} \mathrm{O}$. The RF power during the deep etching is $2000 \mathrm{~W}$, the bias is $-35 \mathrm{~V}$ and the control of temperature is better than $\pm 1{ }^{\circ} \mathrm{C}$. The etching rate depends on the hole size. For 500 $\mu \mathrm{m}$ diameter holes it is estimated to be $7 \mu \mathrm{m} \mathrm{min}^{-1}$. When the front surface of the wafer has been etched down to $13 \mu \mathrm{m}$, $45 \mathrm{~min}$ are then required to realize the membranes from the backside [16, 17].

\section{Optical characterization setup}

The optical measurement setup is illustrated in figure 7. This measurement setup was using the fact that light beams from the outer surface and the hole surface reflect and then travel at different length paths toward the observing screen (in this case the CCD analog video camera).

A photograph of the real configuration is shown in figure 8. The He-Ne laser source has $2 \mathrm{~mW}$ output power (Melles-Griot, USA). ND filter 1 is a variable-rotating ND filter (Melles-Griot, USA), while ND filter 2 had $10 \%$ rate of transmitted light (Edmund Optics, UK). The two ND filters were set such that the intensity of the light beam received by the analog video camera (Fujitsu, Japan) did not saturate its CCD sensors.

The biomimetic strain-sensing microstructure, which resides in a $3 \mathrm{~mm} \times 3 \mathrm{~mm}$ chip, is glued to a $45 \mathrm{~mm} \times 10 \mathrm{~mm} \times$ $512 \mu \mathrm{m}$ Si beam to make a sample that was then held with a clamp (Melles-Griot, USA), held in an XYZ translation stage (Eksma, Lithuania). Bending strain was applied from behind using a millimeter screw (Mitutoyo, Japan). Images captured by the analog video camera were then grabbed and converted into digital form by GrabBee III USB Audio/Video Grabber (VVmer, Taiwan). The digital images were then stored for further processing in a data-acquisition PC (Dell Inspiron, Ireland).

\section{Results and discussions}

The microstructure described in figure 5 is our initial work to mimic the natural campaniform sensillum [9, 15]. Furthermore, fabrication and characterization of these structures will help elucidate the true mechanism of high sensitive strain sensing in the real campaniform sensillum, and confirm the modeling and numerical simulation results. On the basis of the previous report [7], we expected that the strain-concentrating hole is one of the mechanisms used by the natural sensor to amplify strain before transducing it into neurosignals.

Microscope images of the fabricated blind-hole- (i.e. intermediary structure after the completion of the process in figure $6(c)$ ) and membrane-in-recess structures are shown in 

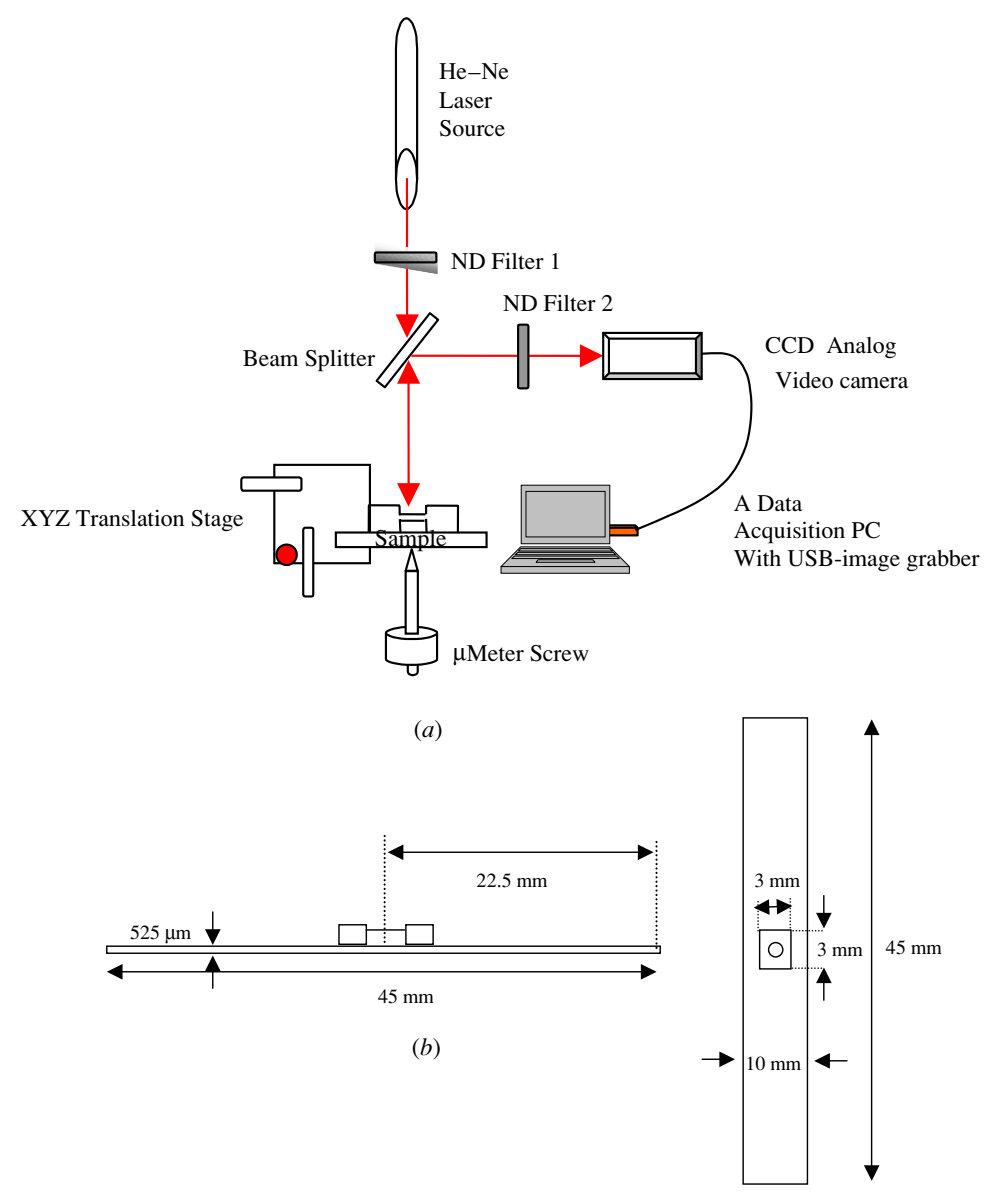

Figure 7. (a) Schematic illustration of the optical measurement setup. (b) Illustrations of the sample showing border conditions. The sample was made by gluing the $3 \times 3 \mathrm{~mm}^{2}$ biomimetic strain-sensing microstructure on top and in the middle of a $45 \mathrm{~mm} \times 10 \mathrm{~mm} \times$ $512 \mu \mathrm{m}$ Si beam.

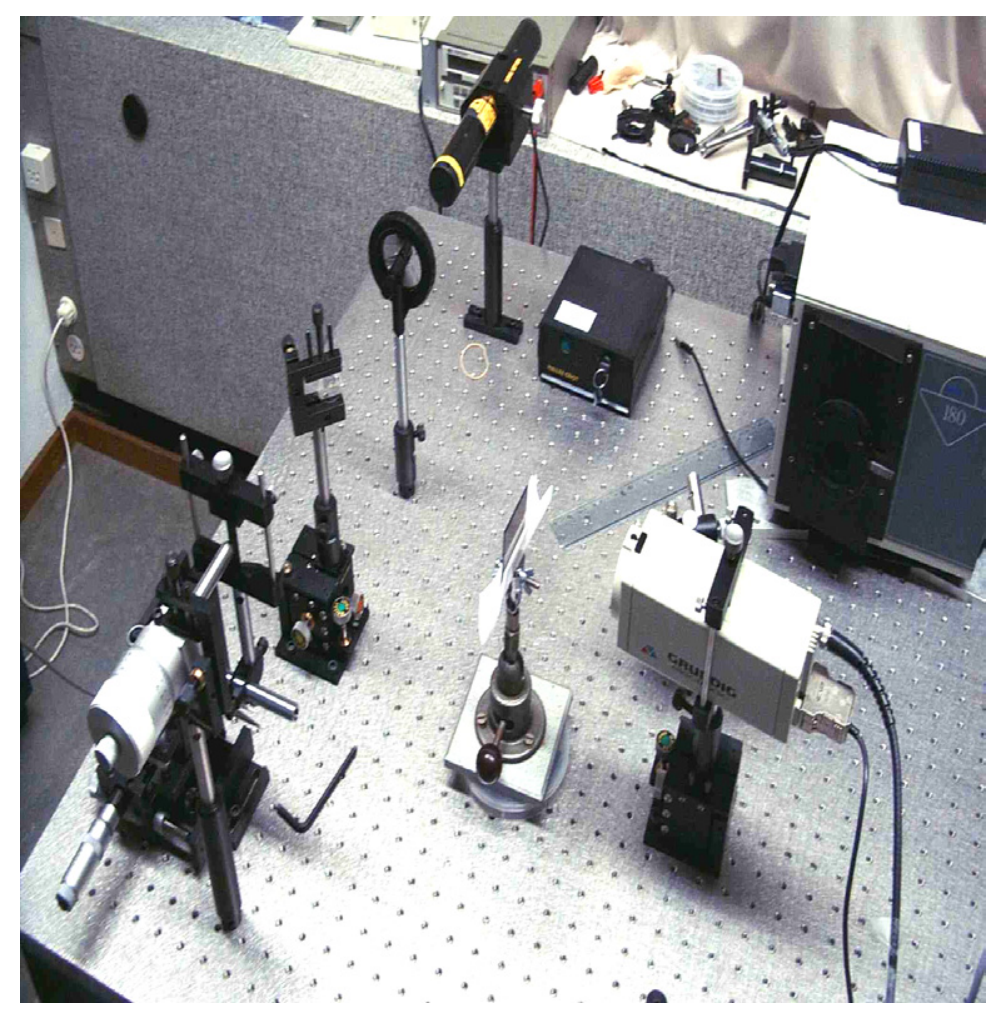

Figure 8. The optical measurement setup. 
(a)

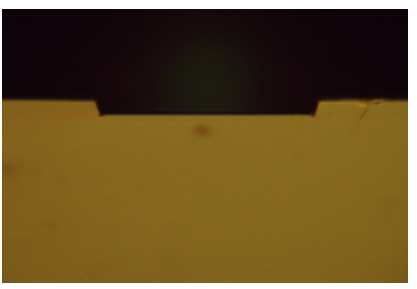

(b)

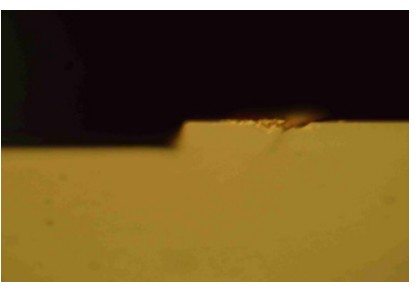

(c)

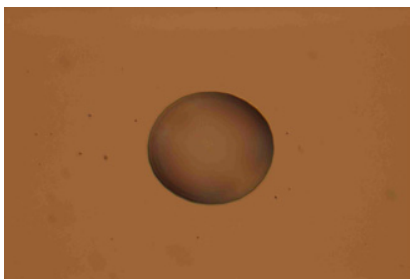

$(d)$

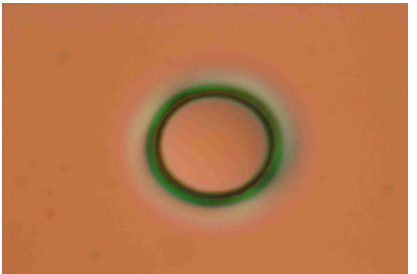

$(e)$

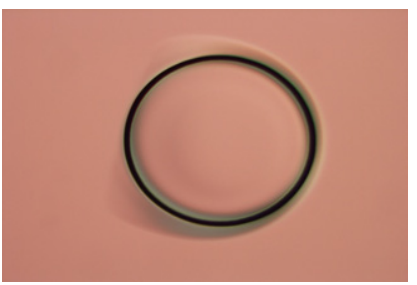

Figure 9. Fabrication results images of the blind-hole structures (after the process shown in figure $6(c)$ ), $(a)$ and $(b)$ : cross-view of a $7 \mu \mathrm{m}$ deep $100 \mu \mathrm{m}$ diameter SiO-SiN coated blind hole, $(c): 5 \mu \mathrm{m}$ deep $25 \mu \mathrm{m}$ diameter bare-Si (prior to $\mathrm{SiO}_{2}-\mathrm{SiN}$ deposition), (d): $5 \mu \mathrm{m}$ deep $25 \mu \mathrm{m}$ diameter coated with $\mathrm{SiO}_{2}-\mathrm{SiN},(e): 5 \mu \mathrm{m}$ deep $200 \mu \mathrm{m}$ diameter $\mathrm{SiO}-\mathrm{SiN}$ covered blind hole.

figures 9 and 10, respectively. The fabrication of the blind-hole structures was quite straightforward and easy. The difference between a $\mathrm{SiO}-\mathrm{SiN}$-coated blind-hole structure (as shown in figures $9(d)$ and $(e)$ ) and bare Si blind-hole structures (shown in figure $9(c)$ ) was also confirmed by optical microscope imaging. The 'ring-corona' structure seen in figure $10(d)$ was probably also caused by slight overetching due to unoptimized time parameter for the backside etching. Comparing with the similar result for the $200 \mu \mathrm{m}$ diameter membrane-in-recess structure (figure 10(c)) without such a 'ring', we know that the process parameter was optimum for 200-250 $\mu$ m diameter. A reduced-backside etching time would probably eliminate this 'ring-corona' structure for higher diameter structures. (a)

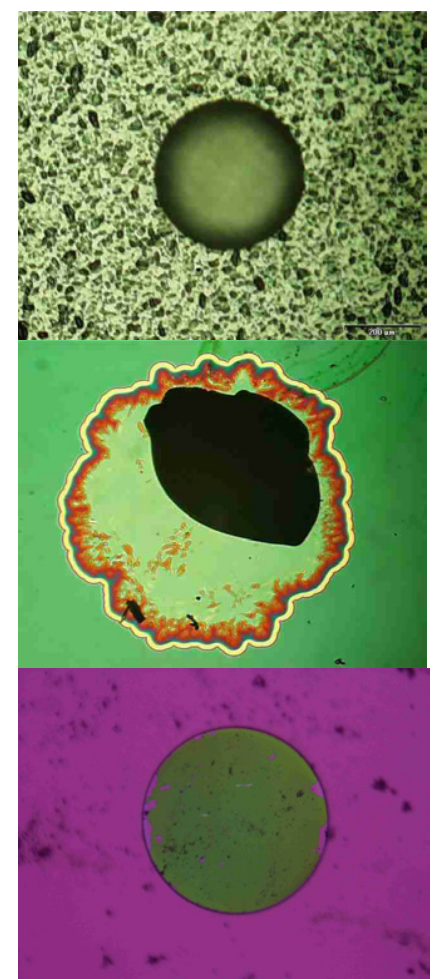

(d)

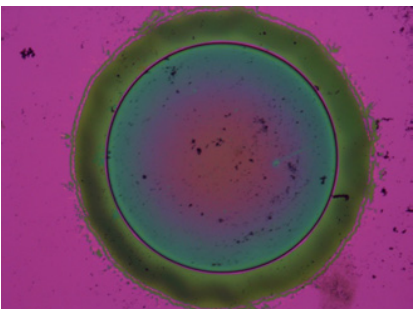

(e)

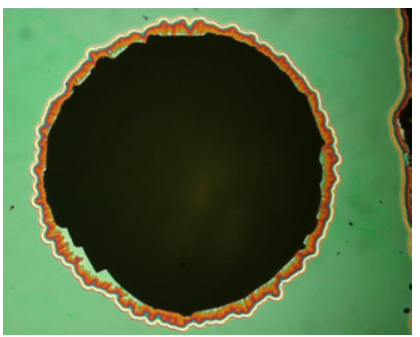

Figure 10. Fabrication results images of the membrane-in-recess structure $13 \mu \mathrm{m}$ deep-front-side-etched (after the completion of the whole process shown in figure 6), (a): $200 \mu \mathrm{m}$ diameter after a backside underetching of $20 \mathrm{~min},(b): 200 \mu \mathrm{m}$ diameter after a backside overetching of $85 \mathrm{~min},(c): 200 \mu \mathrm{m}$ diameter after a time-optimized backside etching, $(d)$ : an intact $500 \mu \mathrm{m}$ diameter structure, (e) $500 \mu \mathrm{m}$ diameter, after an over-backside etching of $85 \mathrm{~min}$.

Figure 11 shows several SEM (scanning electron microscopy) images of the fabricated membrane-in-recess structure. The backside etching process was optimized to make $250 \mu \mathrm{m}$ diameter membrane-in-recess structure. It is clearly shown that over-backside etching was happening to the $500 \mu \mathrm{m}$ diameter sample, simply because it was backside etched too long. The samples of different diameters were made within a single mask such that a particular set of process parameters was only optimum for certain dimensions of the structures. 


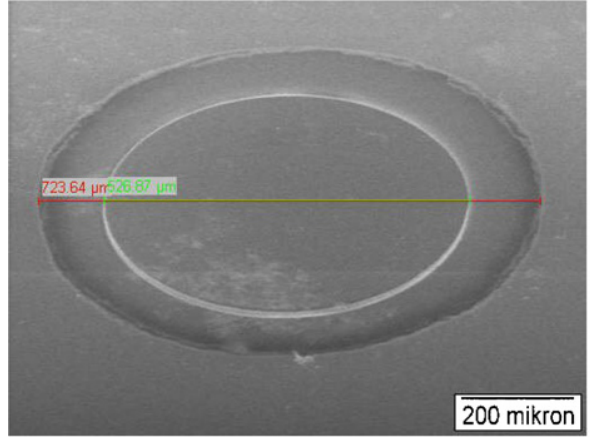

(a)

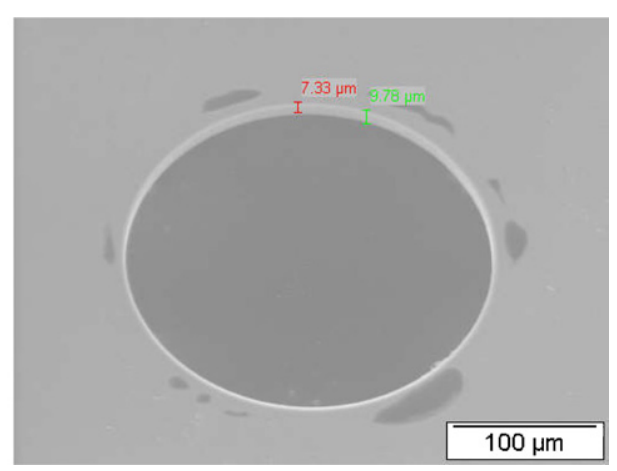

(b)

Figure 11. SEM images of the membrane-in-recess structures $13 \mu \mathrm{m}$ deep-front-side-etched, $(a) 500 \mu \mathrm{m}$ diameter, $(b) 250 \mu \mathrm{m}$ diameter.

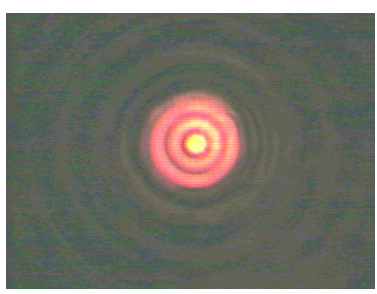

(a)

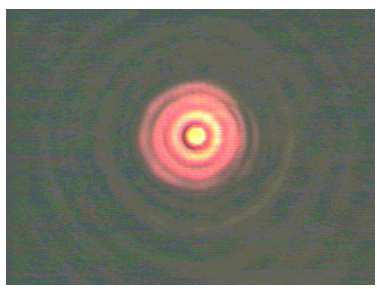

(d)

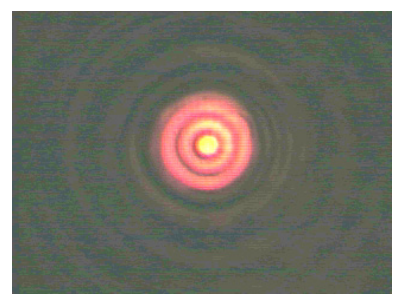

(b)

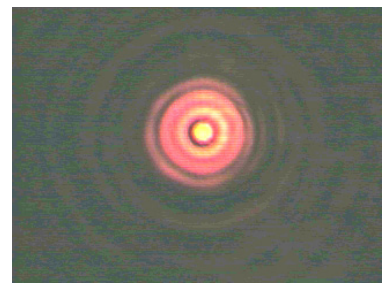

(e)

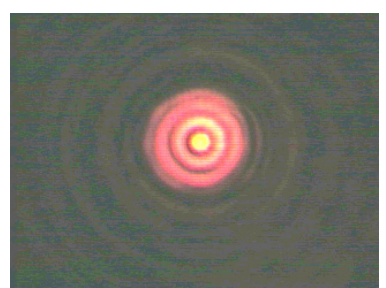

(c)

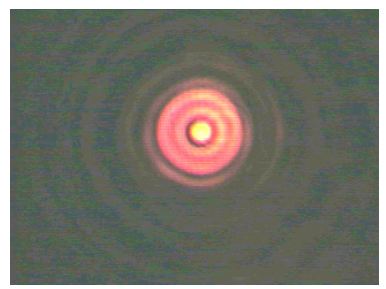

(f)

Figure 12. Images of reflected laser beam from the SiO-SiN membrane-in-recess structure with $13 \mu \mathrm{m}$ depth, $500 \mu \mathrm{m}$ diameter length and $500 \mathrm{~nm}$ total membrane thickness, at different flexural strain stimuli, as applied from behind the Si beam (shown in figure 7) by a micrometer screw with $2 \mu \mathrm{m}$ precision (see figure 4): (a) $0 \mu \mathrm{m},(b)+10 \mu \mathrm{m},(c)+20 \mu \mathrm{m},(d)+30 \mu \mathrm{m},(e)+40 \mu \mathrm{m},(f)+50 \mu \mathrm{m}$.

\section{Observations under stress}

The optical measurement setup explained in the earlier section, 'optical characterization setup', used the fact that light beams from the outer surface and the hole surface reflect and then travel at different length paths toward the observing screen (in this case the CCD analog video camera). Furthermore, a cavity of around $512 \mu \mathrm{m}$ is formed between the $\mathrm{SiO}_{2} / \mathrm{SiN}$ membrane and the $\mathrm{Si}$ beam beneath it. As both the membrane and the $\mathrm{Si}$ beam are partially reflective (with $R_{\mathrm{SiO} 2 / \mathrm{SiN}}=9.3542 \%$, and $R_{\mathrm{si}(100)}=34.7443 \%$, both calculated at $0^{\circ}$ angle of incidence ${ }^{3}$ ), a Fabry-Perot interferogram is formed.

Figures $12(a)-(f)$ show the fringes pattern of the SiO$\mathrm{SiN}$-membrane-in-recess structure just before strain is implied (figure 12(a)), and after relative strains from the position shown in figure 12(a) were being implied on the Si beam where the microstructure is glued (figures $12(b)-(f)$ ), at a maximum strain of $+50 \mu \mathrm{m}$ (figure $12(f)$ ). These images were further centered and cropped at $150 \times 150$ pixel $^{2}$ using Adobe Photoshop 7. These cropped images were further filtered and sliced in the middle for line-intensity profile analysis using

\footnotetext{
3 The reflectance coefficient was calculated using TFCALC software.
}

Image Tools ${ }^{4}$. The processed images are shown in figure 13. The middle horizontal line intensity profile of each image in figure 13 was further processed using a spreadsheet program (Excel), and graphed in figure 14.

As we can see from figures 12 and 13, there appeared a $2 \mathrm{D}$ interference fringes pattern, having three orders of maxima from the middle to the edge of the circular aperture (the diameter from edge to edge of the bright circular fringe resembles the diameter of the membrane, i.e. $500 \mu \mathrm{m})$. We also observed circular fringes outside the perimeter, but with much less intensity, possibly due to the diffraction effect, not of Fabry-Perot interferometry. As we inserted the second lower transmission-rate ND filter (ND filter 2 in figure 7(a)), the outer rings were no longer observed. We suspected that the fringes that came from interference due to the optical cavity formed between the membrane and the Si beam beneath acted as a non-ideal Fabry-Perot interferometer.

The intensity profile of the middle row of the images obtained from different flexural strain stimuli is shown in figure 14. We can see that the changes of the intensity

4 Free image processing software developed at The University of Texas Health Science Center at San Antonio Dental School, downloadable from http://ddsdx.uthscsa.edu/dig/itdesc.html. 


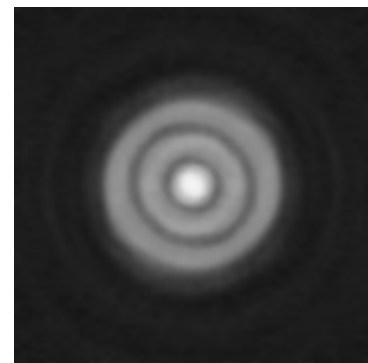

(a)

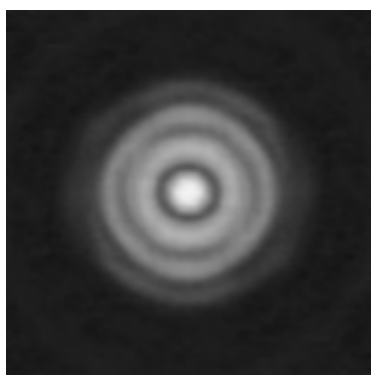

(d)

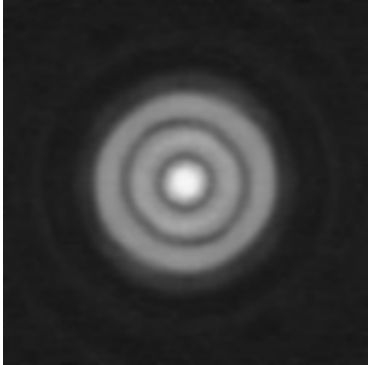

(b)

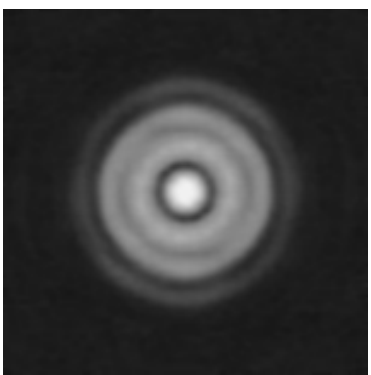

(e)

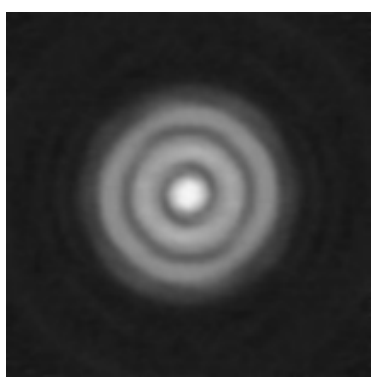

(c)

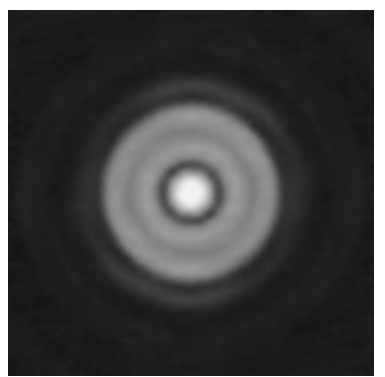

$(f)$

Figure 13. Processed (centered, cropped and filtered) images from figure 12: (a) $0 \mu \mathrm{m},(b)+10 \mu \mathrm{m},(c)+20 \mu \mathrm{m},(d)+30 \mu \mathrm{m}$, $(e)+40 \mu \mathrm{m},(f)+50 \mu \mathrm{m}$.

Response to Strain

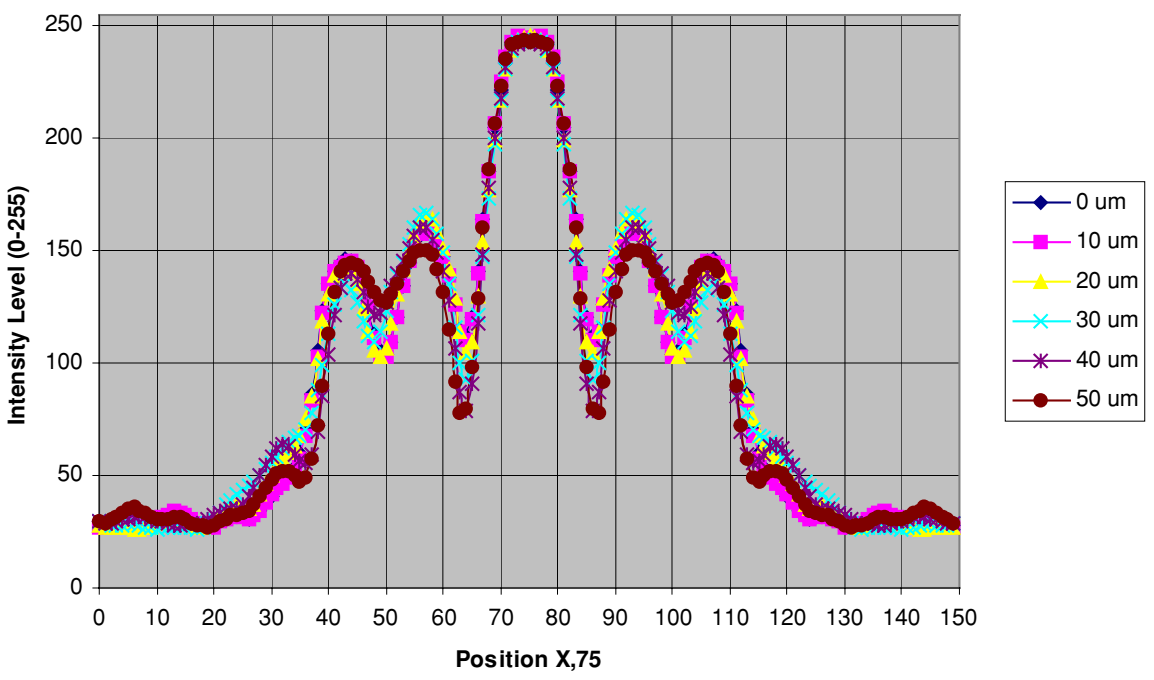

Figure 14. Intensity spatial distribution change as strain is applied.

differ from point to point as a function of spatial location on the membrane. For example the intensity in the center of the membrane (0th-order bright peak) does not change much, relatively calculated, compared to the intensity on the edge of the membrane (third-order bright peak). These differences of relative intensity changes as a function of spatial location within the membrane are shown in figure 15 . These obtained results can be interpreted, among others, from the ideal Fabry-Perot interferometer equation as follows:

$$
\frac{I}{I_{0}}=\frac{1}{1+F \sin ^{2}\left(\frac{2 \pi n d(\cos i)}{\lambda}\right)},
$$

where $I_{0}$ is $(1-a) T^{2} /[1-(1-a) R]^{2}, F$ is finesse $=4(1-$ a) $R /[1-(1-a) R]^{2}, a$ is the absorptivity of the medium, $T$ is the transmittivity of the plates, $R$ is the reflectivity of the plates, $n$ is the index of refraction of the medium, $d$ is the separation of the plates, $i$ is the angle of incidence, and $\lambda$ is wavelength of incident light $633 \mathrm{~nm}$.

We believe that differences shown in figure 15 are due to changes in the sharpness (i.e. $F=$ finesse in the above equation) caused by the applied flexural strain. The finesse is a function of the reflectivity of the plates which in turn is a function of the angle of incidence. As we applied the strain to the structural beam beneath, the membrane did not bend similarly, causing different angles of incidence along different spatial locations on the membrane. For the time being, this non-similar deflection (which is most likely of indentation movement, i.e. downward in the middle area 


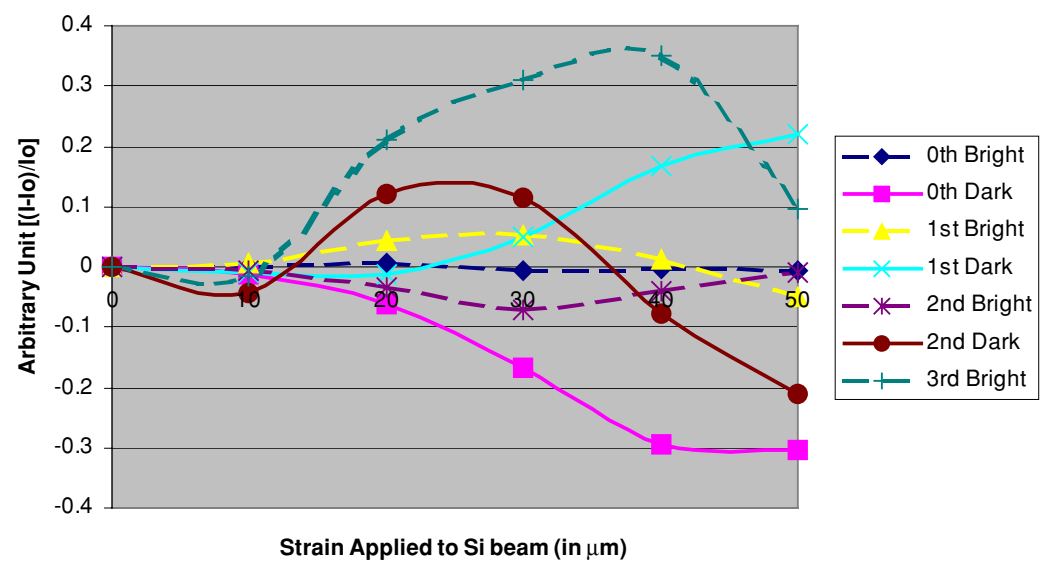

Figure 15. Relative intensity changes in bright and dark peaks of the interferogram as a function of the applied strain. Different peaks show different changes, indicating nonlinear behavior of the deflection, thus a strain-amplifying property.

of the membrane) can be thought of as being associated with the structure's strain-amplifying property as predicted by modeling and finite-element numerical simulation results, shown in previous sections.

Currently, we are trying to interpret this image data in a more quantitative and detailed way, so that we can predict how the membrane moves as flexural strain/stress is applied (see [18] for detail in the discussion of this result).

From the simulation, as well as the optical characterization, we predicted that the blind-hole structure concentrates the strain on its edge. The membrane further amplifies this concentrated strain in the hole, as it deflects non-similarly to the bending of the beam structure beneath it. It is this non-similar deflection of the membrane, as well as the strain-concentrating property of the hole that can be utilized further for developing a new type of high-sensitive MEMS-based strain sensor.

\section{Conclusions}

We have fabricated biomimetic membrane-in-recess structure from Si-based materials. The structure is an initial stage in our effort to develop a new type of strain sensor, inspired from the campaniform sensillum of insects. Numerical simulation as well as experimental optical characterization results show that the blind-hole structure concentrates the strain applied, which is further transduced and amplified as a nonlinear deflection in the membrane-in-recess structure, as confirmed from the intensity spatial distribution change from the optical characterization of the membrane in-recess. We expect that we can utilize these properties for building an improved strain sensor in the future. Further work is currently on-going to interpret the images to understand how the structure behaves in a more quantitative way.

\section{Acknowledgments}

This research is made possible by the grant from The Dutch Technology Foundation, STW (under project DEL 6050), Applied Science Foundation of NWO and the technology programme of Ministry of Economic Affairs, The Netherlands. The authors would like to express its gratitude and thanks to the staff and technicians of DIMES (Delft Institute for Microelectronics and Submicron Technologies) for the fabrication and processes. The authors would also like to thank P Rao for his insightful discussion on Fabry-Perot interferometry and other optical phenomena related to the measurement. The first author would also like to thank A Dall'Acqua for her helpful discussion on the mathematics of optical phenomena.

\section{References}

[1] Csete M E and Doyle J C 2002 Reverse engineering of biological complexity Science 295 1664-9

[2] Vincent J F V and Mann D L 2002 Systematic technology transfer from biology to engineering Phil. Trans. R. Soc. A 360 159-73

[3] Ball P 2001 Life's lessons in design Nature 409 413-6

[4] Barth F G, Humphrey J A C and Secomb T W (ed) 2003 Sensors and Sensing in Biology and Engineering (New York: Springer)

[5] Gnatzy W, Grunert U and Bender M 1987 Campaniform sensilla of Calliphora vicina (Insecta, Diptera). I. Topography Zoomorphology 106 312-9

[6] Grünert U and Gnatzy W 1987 Campaniform sensilla of Calliphora vicina (Insecta, Diptera) II. Typology Zoomorphology 106 320-8

[7] Skordos A, Chan P H, Vincent J F V and Jeronimidis G 2002 A novel strain sensor based on the campaniform sensillum of insects Phil. Trans. R. Soc. A 360 239-53

[8] Zill S N and Seyfarth E-A 1996 Exoskeletal sensors for walking Sci. Am. 275 86-90

[9] Wicaksono D H B, van Duyl W A, Vincent J F V and French P J 2003 Preliminary study on the design of a new micromachined silicon strain sensor based on the Campaniform sensillum of insects Proc. Semiconductor Advances for Future Electronics 2003, SAFE 2003 (Veldhoven) pp 721-5

[10] Bashir R, Gupta A, Neudeck G W, McElfresh M and Gomez R 2000 On the design of piezoresistive silicon cantilevers with stress concentration regions for scanning probe microscopy applications J. Micromech. Microeng. 10 483-91

[11] Yang M, Zhang X, Vafai K and Ozkan C S 2003 High sensitivity piezoresistive cantilever design and optimization 
for analyte-receptor binding J. Micromech. Microeng. 13 $864-72$

[12] Khaled A-R A, Vafai K, Yang M, Zhang X and Ozkan C S 2003 Analysis, control and augmentation of microcantilever deflections in bio-sensing systems Sensors Actuators B 94 $103-15$

[13] Cao L, Kim T S, Mantell S C and Polla D L 2000 Simulations and fabrication of piezoresistive membrane type MEMS strain sensors Sensors Actuators A 80 273-9

[14] Hautamaki C, Zura S, Mantell S C and Polla D L 1999 Experimental evaluation of MEMS strain sensors embedded in composites J. Microelectromech. Syst. 8 272-9

[15] Wicaksono D H B, Pandraud G, Craciun G, Goosen J F L, van Duyl W A, Vincent J F V and French P J 2004 Design and preliminary fabrication of a new micromachined silicon strain sensor based on the campaniform sensillum of insects Proc. Asia-Pacific Conference of Transducers and MicroNano Technology (APCOT MNT 2004) vol 3-1 pp 437-42

[16] Blauw M A, Zijlstra T, Bakker R A and van der Drift E 2000 Kinetics and crystal orientation dependence in high aspect ratio silicon dry etching J. Vac. Sci. Technol. B $\mathbf{1 8}$ $3453-61$

[17] Craciun G, Blauw M, van der Drift E, Sarro P M and French P J 2002 Temperature influence on etching deep holes with $\mathrm{SF}_{6} / \mathrm{O}_{2}$ cryogenic plasma J. Micromech. Microeng. 12 390-4

[18] Wicaksono D H B, Pandraud G and French P J 2004 Simple optical characterisation for biomimetic micromachined silicon strain-sensing structure Proc. SPIE $\mathbf{5 8 5 2} 788-95$ 